\title{
Memory and perfect recall in extensive games
}

\author{
Giacomo Bonanno* \\ Department of Economics, \\ University of California, \\ Davis, CA 95616-8578, USA \\ e-mail: gfbonanno@ucdavis.edu
}

May 2002. Revised, February 2003.

\begin{abstract}
The notion of perfect recall in extensive games was introduced by Kuhn (1953), who interpreted it as "equivalent to the assertion that each player is allowed by the rules of the game to remember everything he knew at previous moves and all of his choices at those moves". We provide a syntactic and semantic characterization of perfect recall
\end{abstract}

${ }^{*}$ I am grateful to two anonymous referees for helpful and constructive comments. A first version of this paper was presented at the fifth conference on Logic and the Foundations of Game and Decision Theory (LOFT5), Torino, June 2002. 
based on two independent notions of memory: (1) memory of past knowledge and (2) memory of past actions.

JEL classification number: C71

\section{Introduction}

The notion of perfect recall in extensive games was introduced by Kuhn (1953), who interpreted it as "equivalent to the assertion that each player is allowed by the rules of the game to remember everything he knew at previous moves and all of his choices at those moves". Several attempts have been made to elucidate the content of the notion of perfect recall: van Benthem (2001) proposes a syntactic interpretation; Okada (1987) and Ritzberger (1999) offer semantic decompositions into independent properties; Kline (2002) clarifies the relationship between perfect recall, ex ante optimality and time consistency. The decompositions of perfect recall that have been offered (see, in particular, Ritzberger, 1999, Theorem 2, p. 81) involve several notions and one is left wondering which of these, if any, can be interpreted as capturing in a precise way the two types of memory traditionally associated with perfect recall, namely memory of past knowledge and memory of past actions. In this paper we integrate the two approaches, syntactic and semantic, and provide a simple decomposition of perfect recall into two properties.

One property, which we call 'memory of past knowledge', is known in the literature. It was introduced in game theory by Okada (1987). Kline (2002) refers to it as 'occurrence memory', while Ritzberger (1999) calls it 'strong ordering'. An essentially identical property, called 'no forgetting', was introduced in the computer science literature by Ladner and Reif (1986) and Halpern and Vardi (1986). We provide a characterization of it in terms of an axiom that explicitly captures the notion of remembering what one knew in the past. This 
is done in Section 3.

The other property, which we call 'action recall', expresses the notion of remembering what one did in the past. It is introduced in Section 4, where a syntactic characterization is provided.

The two notions are shown to be independent: a player can forget what she knew and yet remember what she did in the past or, conversely, she can forget what actions she took in the past but remember what she knew at the time. In Section 5 we show that, together, the two axioms characterize the property of perfect recall.

Okada (1987, Proposition 4, p. 89) shows that, within the class of extensive forms that satisfy 'memory of past knowledge', perfect recall is equivalent to 'complete inflation' (see also Ritzberger, 1999, Theorem 2(b), p. 81). The latter property was introduced by Dalkey (1953). Our result that perfect recall is equivalent to the conjunction of memory of past knowledge and action recall, implies therefore that, within the class of extensive forms that satisfy memory of past knowledge, action recall is equivalent to complete inflation. Thus one aspect of our contribution is that we provide a syntactic characterization of the traditional property of complete inflation and its conventional interpretation in terms of remembering what one did in the past. The relationship between action recall and complete inflation is further explored in Section 6, which is devoted to a discussion of related literature.

The next section introduces definitions and notation.

\section{Notation and definitions}

We use the tree-based definition of extensive game, which is due to Kuhn (1953). Since our analysis deals with the structure of moves and information, and is independent of payoffs, we shall focus on extensive forms and follow closely the definition given by Selten (1975).

The first component of an extensive form is a finite rooted tree $\left\langle T, \rightarrow, t_{0}\right\rangle$ where $t_{0}$ denotes the root and, for any two nodes $t, x \in T, t \rightarrow x$ denotes that $t$ is the immediate predecessor 
of $x$ or $x$ is an immediate successor of $t$. We denote by $\prec$ the transitive closure of $\rightarrow$. Thus $t \prec x$ if there is a path from $t$ to $x$, in which case we say that $t$ is a predecessor of $x$ or $x$ is a successor of $t$. We write $t \precsim x$ as a short-hand for $t=x$ or $t \prec x$. Let $Z$ be the set of terminal nodes, that is, nodes that have no successors and $X=T \backslash Z$ the set of decision nodes.

The second component is a set of players $N=\{0,1, \ldots, n\}$ and a partition $\left\{X_{0}, X_{1}, \ldots, X_{n}\right\}$ of the set of decision nodes $X$. For every player $i \in N, X_{i}$ is the set of decision nodes of player $i$. Players $i=1, \ldots, n$ are called personal players, while player 0 is called Nature and represents events that are not the outcome of actions taken by personal players.

The third component is an equivalence relation $\sim_{i} \subseteq X_{i} \times X_{i}$ for every player $i \in N$ (that is, a binary relation that is reflexive, symmetric and transitive) satisfying the following constraint: if $t, t^{\prime} \in X_{i}$ and $t \sim_{i} t^{\prime}$ then the number of immediate successors of $t$ is equal to the number of immediate successors of $t^{\prime}$. The interpretation of $t \sim_{i} t^{\prime}$ is that player $i$ cannot distinguish between $t$ and $t^{\prime}$, that is, as far as she knows, she could be either at node $t$ or at node $t^{\prime}$. The equivalence classes of $\sim_{i}$ partition $X_{i}$ and are called the information sets of player i.

The fourth, and last, component is, for every player $i \in N$, her choice partition, which, for each of her information sets, partitions the edges out of nodes in that information set (that is, the set of ordered pairs $(t, x)$ such that $t \rightarrow x)$ into player $i$ 's choices at that information set. If $(t, x)$ belongs to choice $c$ we write $t \rightarrow_{c} x$. The choice partition satisfies the following constraints: (1) if $t \rightarrow_{c} x$ and $t \rightarrow_{c} x^{\prime}$ then $x=x^{\prime}$, and (2) if $t \rightarrow_{c} x$ and $t \sim_{i} t^{\prime}$ then there exists an $x^{\prime}$ such that $t^{\prime} \rightarrow_{c} x^{\prime}$. The first condition says that a choice at a node selects a unique immediate successor, while the second condition says that if a choice is available at one node of an information set then it is available at every node in that information set.

In the traditional definition of extensive game further restrictions are imposed: one is known as 'no absent-mindedness' and the other as 'perfect recall'. The definitions will be given below. We do not incorporate these two requirements into the definition of extensive 
form because our purpose is to characterize the class of extensive forms that satisfy them.

An example of an extensive form is given in Figure 1. Here the set of players is $N=$ $\{1,2\}$, the set of terminal nodes is $Z=\left\{z_{1}, \ldots, z_{7}\right\}$ and the set of decision nodes is $X=$ $\left\{t_{0}, t, t^{\prime}, y, x, x^{\prime}\right\}$. The set of player 1's decision nodes is $X_{1}=\left\{t_{0}, y\right\}$, while the set of player 2 's decision nodes is $X_{2}=\left\{t, t^{\prime}, x, x^{\prime}\right\}$. The equivalence relations are $\sim_{1}=\left\{\left(t_{0}, t_{0}\right),(y, y)\right\}$ and $\sim_{2}=\left\{(t, t),\left(t, t^{\prime}\right),\left(t^{\prime}, t\right),\left(t^{\prime}, t^{\prime}\right),(x, x),\left(x, x^{\prime}\right),\left(x^{\prime}, x\right),\left(x^{\prime}, x^{\prime}\right)\right\}$. Thus, for example, player 2 's information sets are $\left\{t, t^{\prime}\right\}$ and $\left\{x, x^{\prime}\right\}$. We use the graphic convention of representing an information set as a rounded rectangle enclosing the corresponding nodes, if there are at least two nodes, while if an information set is a singleton we do not draw anything around it. Furthermore, since all the nodes in an information set belong to the same player, we write the corresponding player only once inside the rectangle. The choices are shown by labeling the corresponding edges in such a way that two edges belong to the same choice if and only if they are assigned the same label. Thus, for example, in Figure $1 x \rightarrow_{g} z_{2}$ and $x^{\prime} \rightarrow_{g} z_{4}$, so that player 2's choice $g$ is $\left\{\left(x, z_{2}\right),\left(x^{\prime}, z_{4}\right)\right\}$. As a further example of our notation, we have that $t \rightarrow x$, that is, $x$ is an immediate successor of $t$, and $t \prec z_{3}$, that is, $z_{3}$ is a successor of $t$. 


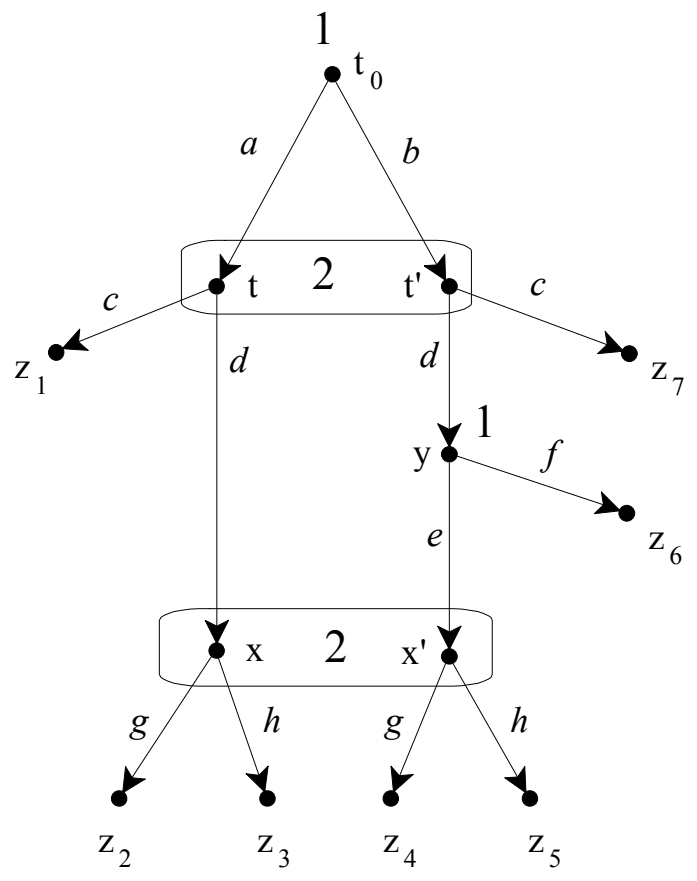

Figure 1

Traditionally, game theorists have restricted attention to games with perfect recall. ${ }^{1}$ This property, which was introduced by Kuhn (1953), requires that if a node $y$ of player $i$ comes after a choice $c$ at a previous node $t$ of player $i$, then every node in the information set that contains $y$ also comes after the same choice $c$ at the information set that contains $t$ (for example, the extensive form of Figure 1 satisfies perfect recall). Formally, ${ }^{2}$

For every player $i$, for all nodes $t, y, y^{\prime} \in X_{i}$ and $x \in T$ and for every choice $c$, if $t \rightarrow_{c} x, x \precsim y$ and $y \sim_{i} y^{\prime}$ then there exist nodes $t^{\prime} \in X_{i}$ and $x^{\prime} \in T$ such

that $t \sim_{i} t^{\prime}, t^{\prime} \rightarrow_{c} x^{\prime}$ and $x^{\prime} \precsim y^{\prime}$.

\footnotetext{
${ }^{1}$ Piccione and Rubinstein (1997) is a notable exception. It gave rise to an entire issue of Games and Economic Behavior (Vol. 20, 1997) being devoted to the consequences of relaxing perfect recall or some of its implications.

${ }^{2}$ The definition given below is Selten's (1975) reformulation of the original definition given by Kuhn in terms of pure strategies.
} 
What does this property mean? Traditionally, as suggested by Kuhn himself, perfect recall has been interpreted as requiring that a player remember what she knew in the past as well as what she did in the past. These are clearly two independent requirements. For example, Figure 2 shows a one-person extensive form where at node $x$ the player remembers what he knew in the past (i.e. at node $t_{0}$ ) but not what choice he made. Conversely, it is possible for a player to remember what actions he took in the past while at the same time not retaining the knowledge he had then (examples will be given in Section 4).

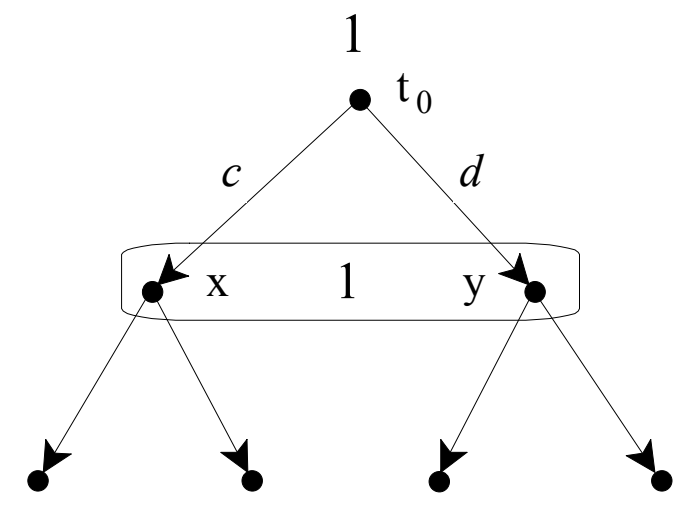

Figure 2

Several papers have been devoted to the study of perfect recall and its decomposition into a number of independent properties (see, in particular, Okada, 1987, Ritzberger, 1999, 2002, and Kline, 2002). In this paper we offer an alternative decomposition and characterization that captures in a precise way the intuitive double requirement on memory: memory of past knowledge and memory of past actions.

We interpret the precedence relation $\prec$ as a temporal relation and associate with it the standard future and past operators from basic temporal logic, denoted by $G$ and $H$ (see, for example, Burgess, 1984, or Goldblatt, 1992). Furthermore, to the equivalence relation $\sim_{i}$ of player $i$ we associate a knowledge operator $K_{i}$. The intended interpretation is as follows: 
$G \phi$ : "it is Going to be the case at every future time that $\phi "$

$H \phi$ : "it Has always been the case that $\phi "$

$K_{i} \phi$ : "player $i$ Knows that $\phi "$.

Note that, in general, the temporal precedence relation $\prec$ defined over the set of nodes does not necessarily induce a temporal precedence relation over the set of information sets. Consider, for example, the extensive game of Figure 3, first proposed by Kuhn (1953). A possible interpretation is as follows: player 1 is an employer and players 2 and 3 are job candidates. When a job candidate (e.g. player 3) receives a job offer, he does not know whether he is the first candidate to whom the offer is made (e.g. the situation is as captured by node $w$ ) or the offer was previously made to the other candidate who turned it down (e.g. the situation is as captured by node $y$ ). Thus at node $y$ it is the case that in the past an offer was made to player 2, but player 3 does not know this (since he considers it possible that he is at node $w$ where the offer made to him is the very first offer). ${ }^{3}$ Consider the information sets $h=\{x, z\}$ and $h^{\prime}=\{y, w\}$. Since $x$ belongs to $h, y$ belongs to $h^{\prime}$ and $x$ temporally precedes $y$, one could tentatively state that $h$ temporally precedes $h^{\prime}$. However, using the same definition, it would follow that $h^{\prime}$ temporally precedes $h$ (since $w \in h^{\prime}$, $z \in h$ and $w$ precedes $z$ ), which would contradict a basic property of temporal precedence, namely asymmetry. Situations like the one depicted in Figure 3 are closely related to the asynchronous systems discussed in the computer science literature (see, for example, Halpern and Vardi, 1986, Fagin et al, 1995, Halpern et al, 2002). In such systems one can associate a time with each node and (some or all) players may not know the time (in the above figure, for example, player 2 does not know whether he is making a choice at time 1, represented by node $x$, or at time 2 , represented by node $z$ ). Synchronous systems, where players always know the time, are closely related to von Neumann games (see Section 6), where the temporal

\footnotetext{
${ }^{3}$ Note that, since the information sets $h=\{x, z\}$ and $h^{\prime}=\{y, w\}$ belong to different players (and none of them is player 1), this game satisfies the definition of perfect recall. If the two information sets $h$ and $h^{\prime}$ belonged to the same player (say, both to player 2) then we would have a situation of imperfect recall where the player does not remember whether he previosly made a choice.
} 
precedence relation over nodes can be extended to the information sets.

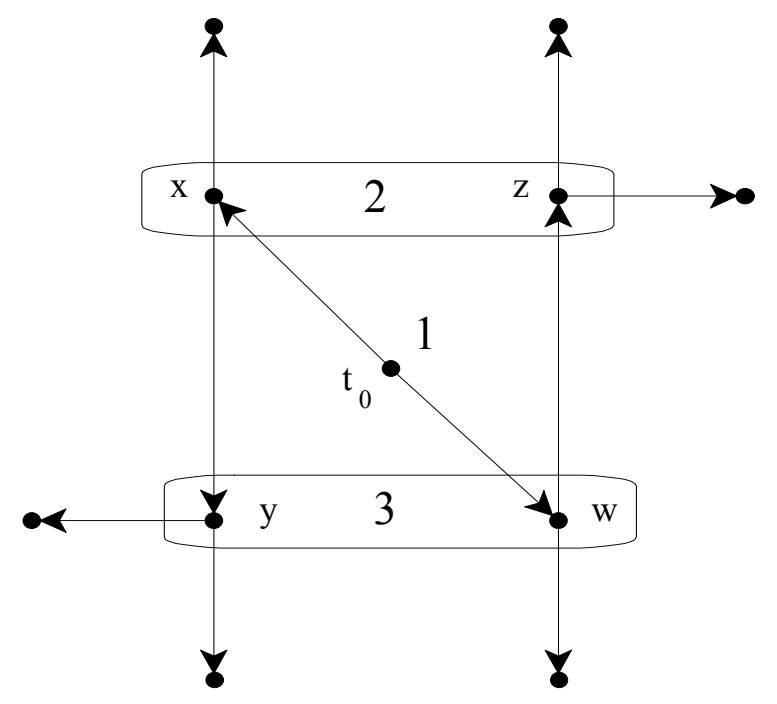

Figure 3

The formal language is built in the usual way from a countable set $S$ of atomic propositions, the connectives $\neg$ (for "not") and $\vee$ (for "or") and the modal operators. ${ }^{4}$ Let $F \phi \stackrel{\text { def }}{=} \neg G \neg \phi$ and $P \phi \stackrel{\text { def }}{=} \neg H \neg \phi$. Thus the interpretation is:

$F \phi$ : "at some Future time it will be the case that $\phi$ "

$P \phi$ : "at some Past time it was the case that $\phi$ ".

Given an extensive form one obtains a model based on it by adding a function $V: S \rightarrow$ $2^{T}$ (where $2^{T}$ denotes the set of subsets of $T$ ) that associates with every atomic proposition $q \in S$ the set of nodes at which $q$ is true. Truth of a formula $\phi$ at a node $t$, denoted by $t \models \phi$, is defined inductively as follows:

if $q$ is an atomic proposition, $t=q$ if and only if $t \in V(q)$,

$t \models \neg \phi$ if and only if $t \not \models \phi$ and $t \models \phi \vee \psi$ if and only if either $t \models \phi$ or $t \models \psi$ (or both), $t \models G \phi$ if and only if $t^{\prime} \models \phi$ for all $t^{\prime}$ such that $t \prec t^{\prime}$,

${ }^{4}$ Thus the set $\Phi$ of formulas is defined inductively as follows: (1) $q \in \Phi$ for every atomic proposition $q \in S$, (2) if $\phi, \psi \in \Phi$ then all of the following belong to $\Phi: \neg \phi, \phi \vee \psi, G \phi, H \phi$ and $K_{i} \phi$. See, for example, Chellas (1984) or Blackburn et al. (2001). The connectives $\wedge$ (for "and") and $\rightarrow$ (for "if ... then") are defined as usual: $\phi \wedge \psi \stackrel{\text { def }}{=} \neg(\neg \phi \vee \neg \psi)$ and $\phi \rightarrow \psi \stackrel{\text { def }}{=} \neg \phi \vee \psi$. 
$t \models H \phi$ if and only if $t^{\prime \prime} \models \phi$ for all $t^{\prime \prime}$ such that $t^{\prime \prime} \prec t$,

$t \models K_{i} \phi$ if and only if $t^{\prime} \models \phi$ for all $t^{\prime}$ such that $t \sim_{i} t^{\prime}$.

Thus $G \phi(H \phi)$ is true at node $t$ if and only if $\phi$ is true at every successor (predecessor) of $t$, while $F \phi(P \phi)$ is true at $t$ if and only if $\phi$ is true at some successor (predecessor) of $t$. Furthermore, $K_{i} \phi$ is true at node $t$ if and only if either $t \notin X_{i}$ (see Remark 2 below) or $t \in X_{i}$ and $\phi$ is true at every node in the information set of player $i$ containing $t$.

Using the above definitions one can thus extend the function $V$ to the set of formulas and denote by $V(\phi)$ the truth set of formula $\phi$, that is, $V(\phi)=\{t \in T: t \models \phi\}$. Throughout the paper we shall consider models where the set of atomic propositions includes, for every $i \in N$, the proposition $\operatorname{turn}_{i}$, whose intended interpretation is "it is player $i$ 's turn to move" and whose truth set, in every model, is $X_{i}$. That is, for every node $t$ and every player $i$, $t \models \operatorname{turn}_{i}$ if and only if $t \in X_{i}$. [The atomic propositions $\operatorname{turn}_{i}(i \in N)$ are the only ones whose truth set is constrained to be the same in every model; the truth set of any other atomic proposition can be different in different models of the same extensive form.]

A formula $\phi$ is valid in a model if $t \models \phi$ for all $t \in T$, that is, if $\phi$ is true at every node. A formula $\phi$ is valid in an extensive form if it is valid in every model based on it.

Definition 1 A property of extensive forms is characterized by an axiom if the axiom is valid in every extensive form that satisfies the property and, conversely, if whenever the axiom is valid in an extensive form then the extensive form satisfies the property.

The "axiomatizations" provided in this paper are characterizations of properties of extensive games by means of axioms, in the sense of the above definition. This is known in modal logic as 'frame definability (or distinguishability)' (see, for example, Blackburn et al., 2001, p. 125 or Halpern, 1998, p. 134). ${ }^{5}$

\footnotetext{
${ }^{5}$ Frame distinguishability is different from the notion of a sound and complete axiomatization in the logician's sense, namely a collection of formulas and inference rules such that every formula provable from this collection is valid in the set of models in question and, conversely, every valid formula is provable from this set. Sound and complete axiomatizations for knowledge and time were first discussed in Halpern and Vardi (1986). A thorough account is given in Halpern et al. (2002).
} 
We conclude this section by drawing attention to a trivial fact (well-known in modal logic: see Chellas, 1984, p. 77), which will be used in some of the proofs.

Remark 2 Fix a player $i$ and a node $t$ that does not belong to player $i$, that is $t \notin X_{i}$. Then, for every formula $\phi$, the formula $K_{i} \phi$ is trivially true at $t$, that $i s, t \models K_{i} \phi$. In fact, for $t \not \models K_{i} \phi$ to be the case there would have to exist a $t^{\prime}$ such that $t \sim_{i} t^{\prime}$ and $t^{\prime} \not \models \phi . B u t \sim_{i}$ is defined only on $X_{i}$ (that is, $\sim_{i}$ is empty-valued on $T \backslash X_{i}$ ).

\section{$3 \quad$ Memory of past knowledge}

In extensive forms the requirement that a player remember what she knew at previous times when it was her turn to move is captured by the following property ('KM' stands for 'Knowledge Memory'), which can be found in Okada (1987, p. 89). ${ }^{6}$ The property says that if $t$ and $y$ are decision nodes of player $i$ and $t$ precedes $y$, then every node $y^{\prime}$ in the information set of player $i$ that contains $y$ has a predecessor in the information set that contains $t$. Formally,

If $t, y \in X_{i}$ and $t \prec y$, then for every $y^{\prime}$ such that $y \sim_{i} y^{\prime}$

there exists a $t^{\prime} \in X_{i}$ such that $t \sim_{i} t^{\prime}$ and $t^{\prime} \prec y^{\prime}$.

Every extensive form with perfect recall clearly satisfies this property. However, there are extensive forms that violate perfect recall and yet satisfy $(K M)$. An example is the extensive form of Figure 2.

The following axiom characterizes property $(K M)\left(M E M_{K}\right.$ stands for 'Memory of Knowledge').

\footnotetext{
${ }^{6}$ Kline (2002, p. 288) refers to it as 'occurrence memory', while Ritzberger (1999, p. 77) calls it 'strong ordering'. An essentially identical property, called 'no forgetting', was discussed by Halpern and Vardi (1986, p. 313) and was later renamed 'perfect recall' (Fagin et al., 1995, p. 129) .
} 


$$
P\left(\text { turn }_{i} \wedge K_{i} \phi\right) \rightarrow K_{i} P \phi
$$

Axiom $\left(M E M_{K}\right)$ says that if at some time in the past it was player $i$ 's turn to move and at that time he knew that $\phi$, then player $i$ knows now that at some time in the past it was the case that $\phi$.

Proposition 3 Property $(K M)$ is characterized by axiom $\left(M E M_{K}\right)$, that is,

(A) the axiom is valid in every extensive form that satisfies $(K M)$, and

(B) if the axiom is valid in an extensive form, then the extensive form satisfies $(K M)$.

Proof. Fix an extensive form that satisfies property $(K M)$ and any model based on it. Let $y$ be a node such that, for some formula $\phi$ and player $i, y \models P\left(\operatorname{turn}_{i} \wedge K_{i} \phi\right)$. Then there exists a node $t$ such that $t \prec y, t \models \operatorname{turn}_{i}$ (that is, $t \in X_{i}$ ) and $t \models K_{i} \phi$. If $y \notin X_{i}$, then $y \models K_{i} P \phi$ trivially (cf. Remark 2). Suppose, therefore, that $y \in X_{i}$. Fix an arbitrary $y^{\prime}$ such that $y \sim_{i} y^{\prime}$. By property $(K M)$, there exists an $t^{\prime}$ such that $t \sim_{i} t^{\prime}$ and $t^{\prime} \prec y^{\prime}$. Since $t \models K_{i} \phi$ and $t \sim_{i} t^{\prime}, t^{\prime}=\phi$. Thus, since $t^{\prime} \prec y^{\prime}, y^{\prime} \models P \phi$. Hence $y \models K_{i} P \phi$.

Conversely, fix an extensive game that violates property $(K M)$. Then there exist a player $i$, nodes $t, y \in X_{i}$ with $t \prec y$, and a node $y^{\prime}$ such that $y \sim_{i} y^{\prime}$ and

$$
\text { for all } t^{\prime} \text { such that } t \sim_{i} t^{\prime}, t^{\prime} \nprec y^{\prime} .
$$

Let $q$ be an atomic proposition and construct a model where the truth set of $q$ is the information set of player $i$ that contains $t$, that is, $V(q)=\left\{t^{\prime} \in T: t \sim_{i} t^{\prime}\right\} .{ }^{7}$ Then $t=K_{i} q$. Since $t \in X_{i}, t \models$ turn $_{i}$ and, since $t \prec y, y \models P\left(\right.$ turn $\left._{i} \wedge K_{i} q\right)$. By (1), $y^{\prime} \not \models P q$. Hence, since $y \sim_{i} y^{\prime}, y \not \models K_{i} P q$. Thus axiom $\left(M E M_{K}\right)$ is falsified at $y$.

One might wonder whether it is possible to replace the consequent $K_{i} P \phi$ in axiom $\left(M E M_{K}\right)$ with the more explicit statement that the player knows that in the past he knew

\footnotetext{
${ }^{7}$ In line with the modal logic literature, when establishing the correspondence between an axiom and a property of a structure (in our case, an extensive form) the content of the atomic propositions is immaterial. However, one could constrain the construction of models by using only atomic propositions that can be meaningfully interpreted as statements about the extensive form under consideration. The simplest way to do this is to define, for every node $t$ an atomic proposition $q_{t}$ interpreted as "the play of the game is currently at node $t^{\prime \prime}$, so that the truth set of $q_{t}$ would be precisely $\{t\}$. Then for every set of nodes $S=\left\{t_{1}, \ldots, t_{n}\right\}$ there is a formula $\phi_{S}$ whose truth set is $S$, namely the disjunctive formula $\left(q_{t_{1}} \vee \ldots \vee q_{t_{n}}\right)$. If one were to follow this approach, the atomic proposition $q$ in the proof would be replaced by the disjunctive formula whose truth set is the information set of player $i$ that contains $t$.
} 
$\phi$ : $K_{i} P K_{i} \phi$. In other words, one might wonder if in Proposition 3 it is possible to replace axiom $\left(M E M_{K}\right)$ with the following axiom:

$$
P\left(\operatorname{turn}_{i} \wedge K_{i} \phi\right) \rightarrow K_{i} P K_{i} \phi
$$

The answer is negative. In fact, while it is still the case that property $(K M)$ validates axiom $\left(M E M_{K}^{\prime}\right)$, the converse is not true: Figure 4 shows an extensive form where $\left(M E M_{K}^{\prime}\right)$ is valid and yet property $(K M)$ is violated. Let $\phi$ be any formula that is true at node $t$. Then player 2 knows $\phi$ at $t$. At node $t_{0}$ it is trivially true that player 2 knows $\phi$ (cf. Remark 2). Hence $P K_{2} \phi$ is true at both $y$ and $y^{\prime}$. Thus axiom $\left(M E M_{K}^{\prime}\right)$ is satisfied at node $y$, although the extensive form violates property $(K M){ }^{8}$

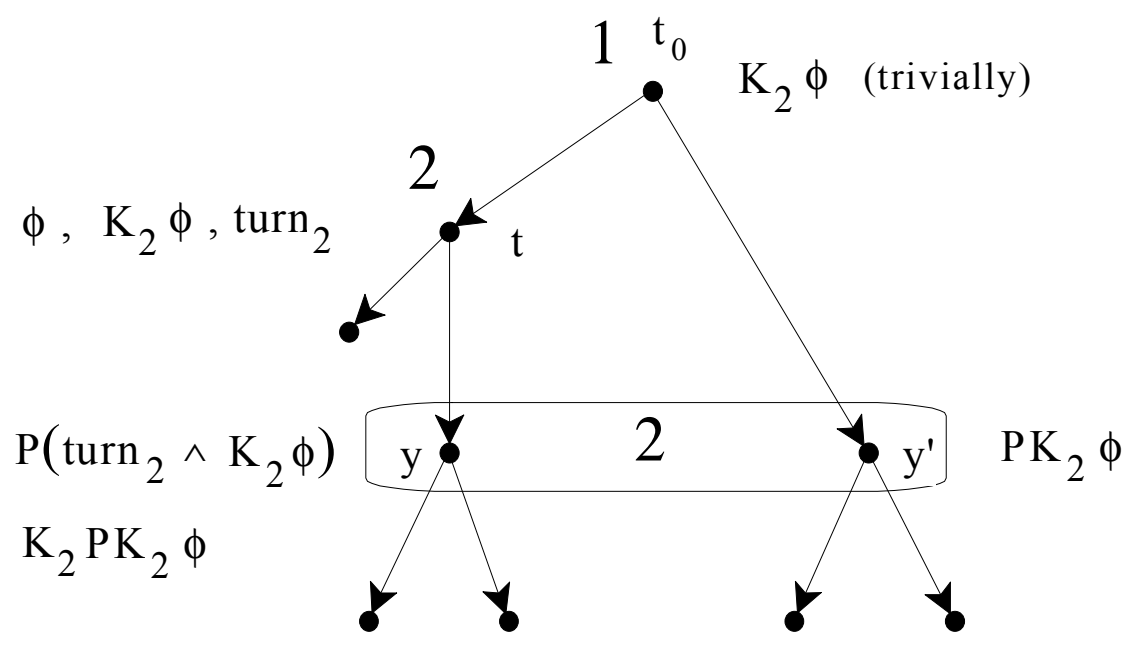

Figure 4

It is possible, however, to obtain a characterization in terms of axiom $\left(M E M_{K}^{\prime}\right)$ by extending the equivalence relation of player $i$ from the set $X_{i}$ to the entire set $T^{9}$ In

\footnotetext{
${ }^{8}$ The fact that axiom $\left(M E M_{K}^{\prime}\right)$ is satisfied at node $y$ is not sufficient to conclude that it is satisfied at every node, that is, that it is valid in the extensive form. However, it is straightforward to check that the only node where the axiom could be falsified for player 2 is indeed node $y$, because that is the only decision node where Pturn $_{2}$ is true. On the other hand, the axiom is clearly valid for player 1 , since the only node that belongs to player 1 is the root and at every other node player 1 trivially knows every formula.

${ }^{9}$ That is, we need to define an equivalence relation $R_{i} \subseteq T \times T$ satisfying the property that if $x \in X_{i}$
} 
such a framework one can also extend property $(K M)$ to the set of all nodes (i.e. drop the restriction that $\left.t, y \in X_{i}\right)$ and show that such an extension of $(K M)$ is characterized by the axiom $P K_{i} \phi \rightarrow K_{i} P K_{i} \phi$ (thus dropping turn $_{i}$ from the antecedent of $\left(M E M_{K}^{\prime}\right)$ ). This is shown in Bonanno (2001), where it is also proved that, if an (extended) extensive form satisfies the property that a player never forgets what she knew in the past, then the extensive form is von Neumann (following the terminology of Kuhn, 1953; p. 52 of reprint in Kuhn, 1997). An extensive game is von Neumann if, whenever $t$ and $x$ are decision nodes of player $i$ that belong to the same information set of player $i$ (that is, $t, x \in X_{i}$ and $t \sim_{i} x$ ), the number of predecessors of $t$ is equal to the number of predecessors of $x$.

A property that has traditionally been incorporated in the definition of extensive game (see Kuhn, 1953 and Selten, 1975) is that no two nodes in an information set of a player be such that one is a predecessor of the other. Violation of this property has been called "absent-mindedness" (see Piccione and Rubinstein, 1997).

Definition 4 Player $i$ is absent-minded at node $y$ if $y \in X_{i}$ and there exists a $t \in X_{i}$ such that $t \sim_{i} y$ and $t \prec y$.

The following lemma says that if property $(K M)$ is satisfied then absent-mindedness "propagates into the past".

Lemma 5 Fix an arbitrary extensive form that satisfies property $(K M)$. Then the following is true for every node $y$ and every player $i$ : if player $i$ is absent-minded at node $y$ then there exists a $t \in X_{i}$ such that $t \prec y$ and player $i$ is absent-minded at $t$.

Proof. Suppose that player $i$ is absent-minded at node $y \in X_{i}$. Then there exists a $t \in X_{i}$ such that $t \sim_{i} y$ and $t \prec y$. Since $\sim_{i}$ is an equivalence relation, $y \sim_{i} t$. Thus, by $(K M)$ (letting $y^{\prime}=t$ ), there exists a $t^{\prime} \in X_{i}$ such that $t \sim_{i} t^{\prime}$ and $t^{\prime} \prec t$. Since $t \sim_{i} t^{\prime}$, by definition of equivalence relation, $t^{\prime} \sim_{i} t$. Thus player $i$ is absent-minded at $t$.

then, for all $y \in T, x R_{i} y$ if and only if $x \sim_{i} y$. In other words, the restriction of $R_{i}$ to $X_{i}$ coincides with $\sim_{i}$, so that the original information sets are preserved. 
With the help of the above lemma, the following proposition is easily proved.

Proposition 6 Property (KM) rules out absent-mindedness.

Proof. Suppose that there is a node $y_{1}$ at which player $i$ is absent-minded. By Lemma 5 there is an infinite sequence $\left\langle y_{1}, y_{2}, \ldots\right\rangle$ in $X_{i}$ such that, for all $k \geq 1, y_{k+1} \prec y_{k}$ and at $y_{k+1}$ player $i$ is absent-minded. Since a tree has no cycles, it follows that for all $j, k \geq 1$ with $j \neq k, y_{j} \neq y_{k}$. But this is not possible, since the number of nodes is finite.

\section{Memory of past actions}

Property $(K M)$ and its characterizing axiom $\left(M E M_{K}\right)$ capture the notion of remembering what one knew in the past. As the example of Figure 2 shows, memory of past knowledge does not imply memory of past actions: an individual can remember what she knew in the past while at the same time forgetting what she did. In this section we want to capture the other component of perfect recall, namely remembering what one did in the past. Furthermore, we want this type of memory to be separate and independent from memory of past knowledge. Intuitively, this is indeed the case: one can remember doing something while at the same time forgetting what he knew at the time. Consider for example the maze shown in Figure 5 and the following situation: an individual (player 1) is given the map of the maze and taken (by player 0) to one of the entrances (either A or B). He is told at which entrance he is. He then enters the maze, turns right at the first junction and left at the second junction. At this stage, perhaps because he is tired, he forgets where he entered the maze. He remembers knowing it at the time and remembers having taken first a right turn and then a left turn. Hence he knows that he is either at junction $x$ or at junction $y$, but this is all he knows. Thus we have a situation where the individual remembers what he did (he first turned right and then left) but forgets what he knew (the entrance at which he started). 


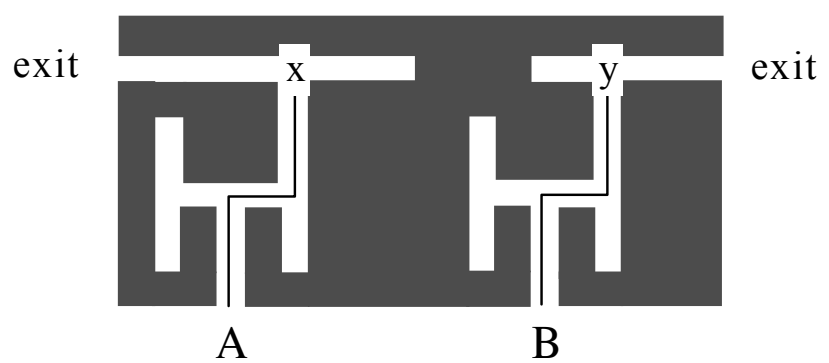

Figure 5

How can we capture a situation like this in an extensive form? The only way we can do this is by separating the notion of action from that of choice. Recall that a choice of a player is a set of edges out of an information set of that player. Thus choices are, by definition, tied to the state of information of the player: remembering a choice necessarily involves remembering the information set at which it was made and, therefore, the knowledge one had at the time. Actions, on the other hand, are a more general notion than choices. One can take the same action, e.g. turn right, in different states of knowledge (i.e. at different information sets). That is, different choices can be considered as instances of the same action. To capture the distinction between actions and choices we need to make an addition to the definition of extensive form, namely we need to add a set of actions (or action labels) $A$ and a function $\alpha(\cdot)$ from choices to actions that assigns an action label to every choice, with two constraints: (1) if $c$ and $c^{\prime}$ are two different choices at the same information set of some player $i$ then they are assigned different action labels, that is, if $t \sim_{i} t^{\prime}, t \rightarrow_{c} x$, $t^{\prime} \rightarrow c^{\prime} x^{\prime}$, and $c \neq c^{\prime}$, then $\alpha(c) \neq \alpha\left(c^{\prime}\right)$, and (2) two choices taken sequentially by the same player at different information sets are also assigned different labels, that is, if $t, y \in X_{i}$ with $t \varkappa_{i} y$ and $t \rightarrow_{c} x, x \precsim y$ and $y \rightarrow_{c^{\prime}} w$ then $\alpha(c) \neq \alpha\left(c^{\prime}\right){ }^{10}$ An extensive form with such an addition will be called an action-labeled extensive form. Every "standard" extensive form, as

\footnotetext{
${ }^{10}$ The requirement that sequential choices by the same player at different information sets be assigned different labels is merely for convenience. Without such a requirement one would have to make the syntax more complex by making it possible to express how many times a particular action was performed in the past (e.g. turning left two times). Nothing would be gained by adding this extra complexity.
} 
defined in Section 2, can be trivially viewed as an action-labeled extensive form where any two different choices are assigned different labels; indeed, when one draws an extensive form one automatically assigns labels to choices in order to identify them. In general, however, in an action-labeled extensive form the set of actions yields a coarsening of the choice partition.

An example of an action-labeled extensive form is shown in Figure 6 and it represents the situation described above for the maze of Figure 5. The set of player 1's actions is $A=\left\{\right.$ left $_{1}$, right $_{1}$, left $_{2}$, right $_{2}$, left $_{3}$, right $\left._{3}\right\}$, where left $t_{1}$ is the action of turning left at the first junction, right $t_{2}$ is the action of turning right at the second junction, etc. The same action can be associated with different information sets: for example, the action right corresponds to one of the choices at node $t$ and also to one of the choices at node $t^{\prime}$, despite the fact that $t$ and $t^{\prime}$ belong to different information sets.

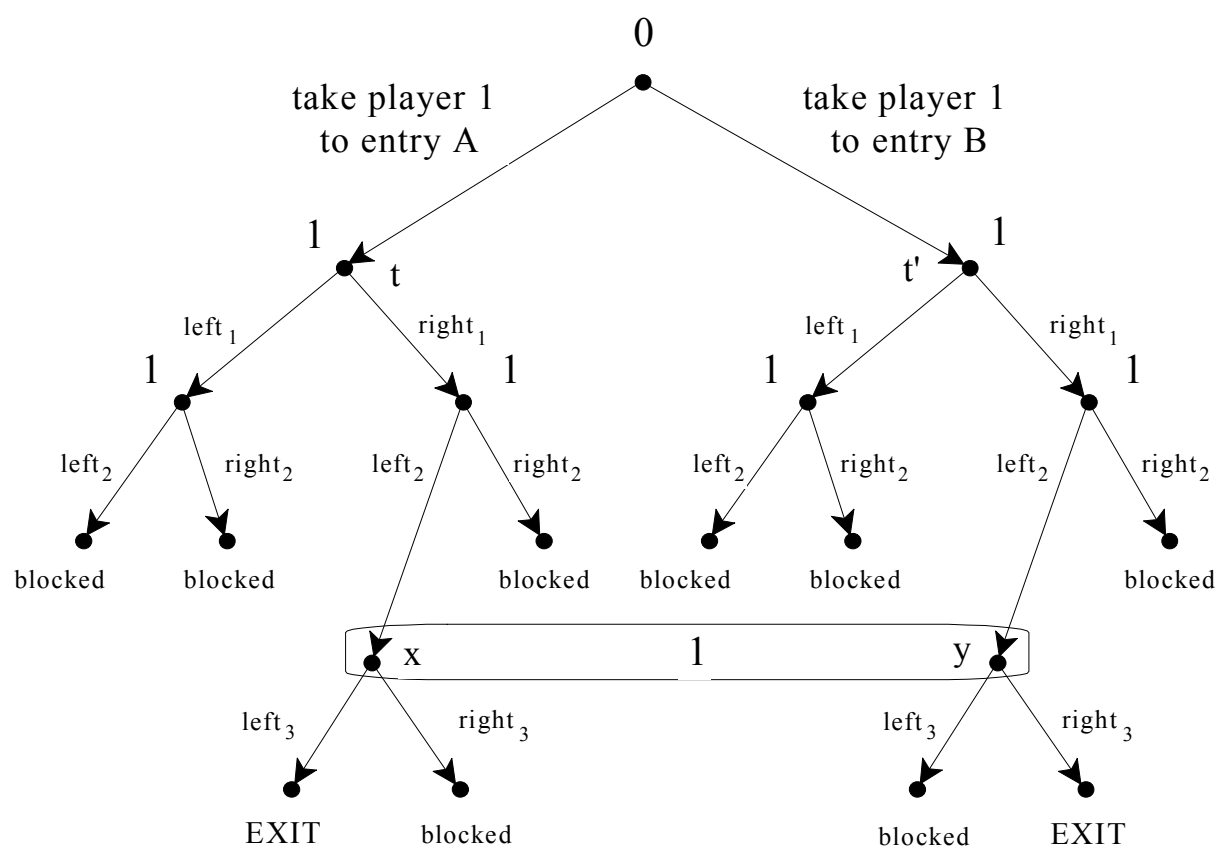

Figure 6

Looking at Figure 6 it is intuitively clear that at, say, node $x$ the individual remembers what he did (he first turned right and then left), but does not know whether he is at junction $x$ 
or at junction $y$ because he has forgotten what he previously knew (and remembers knowing) namely at which entrance he started.

We now define a property and a characterizing axiom that capture the notion of remembering past actions ('AR' stands for 'Action Recall').

Let $t, y \in X_{i}$ and $x \in T$ be such that $t \rightarrow_{c} x, \alpha(c)=a$ and $x \precsim y$.

Then for every $y^{\prime}$ such that $y \sim_{i} y^{\prime}$, there exist a $t^{\prime} \in X_{i}$, a choice

$c^{\prime}$ at $t^{\prime}$ and an $x^{\prime} \in T$ such that $t^{\prime} \rightarrow_{c^{\prime}} x^{\prime}, \alpha\left(c^{\prime}\right)=a$ and $x^{\prime} \precsim y^{\prime}$.

Property $(A R)$ says that if $t$ is a decision node of player $i$ and $x$ is the immediate successor of $t$ following choice $c$ which corresponds to action $a\left(t \in X_{i}, t \rightarrow_{c} x\right.$ and $\left.\alpha(c)=a\right)$ and $y$ is another decision node of player $i$ which is $x$ itself or a successor of $x\left(y \in X_{i}\right.$ and $\left.x \precsim y\right)$ then, for every node $y^{\prime}$ in the information set of player $i$ that contains node $y\left(y \sim_{i} y^{\prime}\right)$, there exist a decision node $t^{\prime}$ of player $i$ and a choice $c^{\prime}$ at $t^{\prime}$ such that the immediate successor $x^{\prime}$ of $t^{\prime}$ following choice $c^{\prime}\left(t^{\prime} \in X_{i}, t^{\prime} \rightarrow_{c^{\prime}} x^{\prime}\right)$ is either $y^{\prime}$ itself or a successor of $y^{\prime}\left(x^{\prime} \precsim y^{\prime}\right)$ and, furthermore, also choice $c^{\prime}$ corresponds to action $a\left(\alpha\left(c^{\prime}\right)=a\right)$. Note that we do not require that $t \sim_{i} t^{\prime}$, otherwise we would be back to the property of perfect recall. Indeed, $(A R)$ does not imply perfect recall. For example, the extensive form of Figure 6 satisfies $(A R)$ but violates perfect recall. 


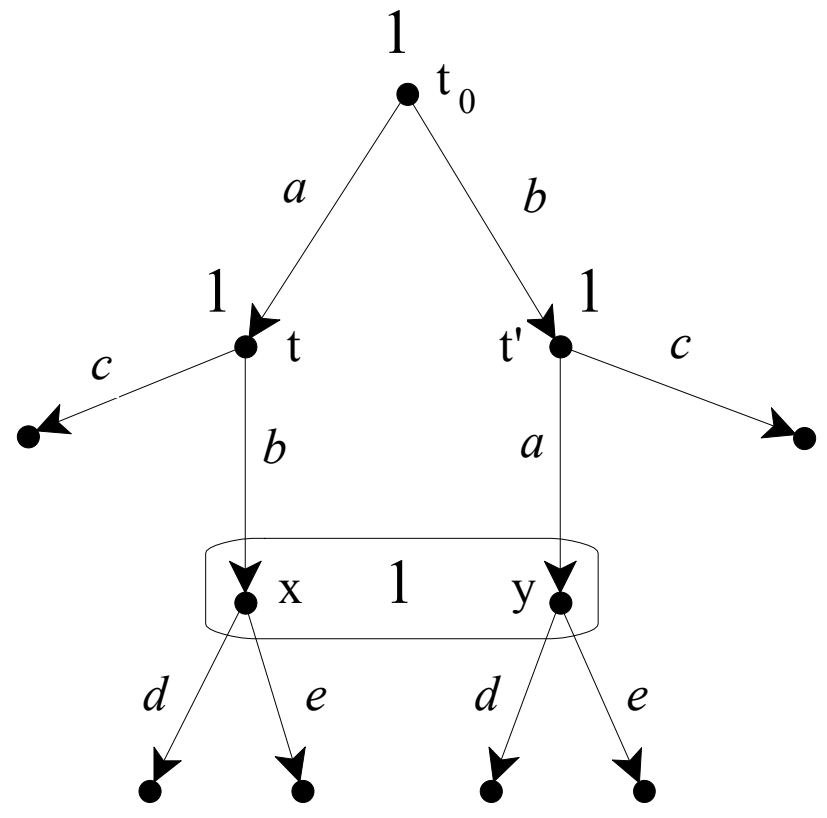

Figure 7

Remark $7(A R)$ requires that at any two nodes in the same information set of player $i$, the player remember all the actions that he took up to that point. Note, however, that $(A R)$ does not require that the player remember the order in which he took those actions. For example, the extensive form of Figure 7 satisfies $(A R)$ but the player does not remember whether he first took action $a$ and then action $b$ or vice versa (although he remembers taking both action a and action b). ${ }^{11}$

In order to characterize memory of actions syntactically, we need to add, for every action $a \in A$ a modal operator $\square_{a}$. The intended interpretation of $\square_{a} \phi$ is "after action $a$ it will be the case that $\phi "$. We also introduce the inverse operator $\square_{a}^{-1}$ so that the interpretation of $\square_{a}^{-1} \phi$ is "before action $a$ it was the case that $\phi$ ". Thus the truth conditions are as follows:

$$
t \models \square_{a} \phi \text { if } x \models \phi \text { whenever, for some choice } c, t \rightarrow_{c} x \text { and } \alpha(c)=a \text {, }
$$

\footnotetext{
${ }^{11}$ For example, after getting up very early in the morning an individual might remember shaving (action a) and brushing his teeth (action b) but be unable to recall whether he first shaved and then brushed his teeth or the other way round.
} 


$$
x \models \square_{a}^{-1} \phi \text { if } t \models \phi \text { whenever, for some choice } c, t \rightarrow_{c} x \text { and } \alpha(c)=a \text {. }
$$

Let $\nabla_{a}$ be the dual of $\square_{a}$ and $\diamond_{a}^{-1}$ the dual of $\square_{a}^{-1}$. That is, $\diamond_{a} \phi \stackrel{\text { def }}{=} \neg \square_{a} \neg \phi$ and $\diamond_{a}^{-1} \phi \stackrel{\text { def }}{=} \neg \square_{a}^{-1} \neg \phi$. Hence the truth conditions are:

$t \models \diamond_{a} \phi$ if there exist a node $x$ and a choice $c$ such that $t \rightarrow_{c} x, \alpha(c)=a$, and $x \models \phi$. $x \models \diamond_{a}^{-1} \phi$ if there exist a node $t$ and a choice $c$ such that $t \rightarrow_{c} x, \alpha(c)=a$, and $t \models \phi{ }^{12}$

Consider the following axiom $\left(M E M_{A}\right.$ stands for 'Memory of Actions'):

$$
\diamond_{a}^{-1} \text { turn }_{i} \rightarrow K_{i}\left(\diamond_{a}^{-1} \text { turn }_{i} \vee P \diamond_{a}^{-1} \text { turn }_{i}\right) \wedge G K_{i}\left(\diamond_{a}^{-1} \text { turn }_{i} \vee P \diamond_{a}^{-1} \text { turn }_{i}\right)
$$

The formula $\diamond_{a}^{-1}$ turn $_{i}$ says that action $a$ was just taken and before action $a$ it was player $i$ 's turn to move, that is, that player $i$ just took action $a$. Thus axiom $\left(M E M_{A}\right)$ says that if player $i$ just took action $a$, then he knows that either he just took action $a$ or that some time in the past he took action $a$ and, furthermore, he will always know this.

Note that the antecedent of $\left(M E M_{A}\right)$ does not contain any hypothesis about what the player knew when he acted. This is the reason why axiom $\left(M E M_{A}\right)$ captures merely memory of past actions and allows a player to forget what he knew in the past, as in the extensive forms of Figures 6 and 7, which satisfy $(A R)$ but not $(K M)$.

Proposition 8 Property $(A R)$ is characterized by axiom $\left(M E M_{A}\right)$, that is,

(A) the axiom is valid in every action-labeled extensive form that satisfies $(A R)$, and

(B) if the axiom is valid in an action-labeled extensive form, then the extensive form satisfies $(A R)$.

\footnotetext{
${ }^{12}$ By definition of action-labeled extensive form, two choices out of the same node must be assigned different action labels, that is, if $t \rightarrow_{c} x$ and $t \rightarrow_{c^{\prime}} x^{\prime}$ with $x \neq x^{\prime}$, then $\alpha(c) \neq \alpha\left(c^{\prime}\right)$. It follows that, for every action $a$ and formula $\phi$, the formula $\nabla_{a} \phi \rightarrow \square_{a} \phi$ is valid in every action-labeled extensive form and, by definition of tree, the same is true of $\diamond_{a}^{-1} \phi \rightarrow \square_{a}^{-1} \phi$. The converse, however, is not true, that is, neither $\square_{a} \phi \rightarrow \nabla_{a} \phi$ nor $\square_{a}^{-1} \phi \rightarrow \diamond_{a}^{-1} \phi$ are valid. In fact, validity of $\square_{a} \phi \rightarrow \diamond_{a} \phi$ would require that for every $t$ there be $c$ and $x$ such that $t \rightarrow_{c} x$ and $\alpha(c)=a$.
} 
Proof. Fix an action-labeled extensive form that satisfies property $(A R)$. Fix arbitrary $x \in T, i \in N$ and $a \in A$ and suppose that $x \models \diamond_{a}^{-1}$ turn $_{i}$. Let $t$ be the immediate predecessor of $x$. Then $t \in X_{i}$ and $t \rightarrow_{c} x$ for some choice $c$ with $\alpha(c)=a$. First we show that $x \models K_{i}\left(\diamond_{a}^{-1}\right.$ turn $_{i} \vee P \diamond_{a}^{-1}$ turn $\left._{i}\right)$. If $x \notin X_{i}$ then it is trivially true (see Remark 2). Suppose therefore that $x \in X_{i}$. Fix an arbitrary $y^{\prime}$ such that $x \sim_{i} y^{\prime}$. Then by $(A R)$ there exist $t^{\prime} \in X_{i}$, a choice $c^{\prime}$ and a node $x^{\prime}$ such that $t^{\prime} \rightarrow_{c^{\prime}} x^{\prime}, \alpha\left(c^{\prime}\right)=a$ and $x^{\prime} \precsim y^{\prime}$. Thus $x^{\prime} \models \diamond_{a}^{-1}$ turn $_{i}$ and $y^{\prime} \models \diamond_{a}^{-1}$ turn $_{i} \vee P \diamond_{a}^{-1}$ turn $_{i}$. Hence $x \models K_{i}\left(\diamond_{a}^{-1}\right.$ turn $_{i} \vee P \diamond_{a}^{-1}$ turn $\left._{i}\right)$. Next we show that $x \models G K_{i}\left(\diamond_{a}^{-1}\right.$ turn $_{i} \vee P \diamond_{a}^{-1}$ turn $\left._{i}\right)$. Fix an arbitrary $y$ such that $x \prec y$. If $y \notin X_{i}$ then $y=$ $K_{i}\left(\diamond_{a}^{-1}\right.$ turn $_{i} \vee P \diamond_{a}^{-1}$ turn $\left._{i}\right)$ trivially (see Remark 2). Suppose therefore that $y \in X_{i}$. Fix an arbitrary $y^{\prime}$ such that $y \sim_{i} y^{\prime}$. Then by $(A R)$ there exist $t^{\prime} \in X_{i}$, a choice $c^{\prime}$ and a node $x^{\prime}$ such that $t^{\prime} \rightarrow c^{\prime} x^{\prime}, \alpha\left(c^{\prime}\right)=a$ and $x^{\prime} \precsim y^{\prime}$. Thus $x^{\prime} \models \diamond_{a}^{-1}$ turn $_{i}$ and $y^{\prime}=\diamond_{a}^{-1}$ turn $_{i} \vee P \diamond_{a}^{-1}$ turn $_{i}$ and $y=K_{i}\left(\diamond_{a}^{-1}\right.$ turn $_{i} \vee P \diamond_{a}^{-1}$ turn $\left._{i}\right)$. Hence $x \models G K_{i}\left(\diamond_{a}^{-1}\right.$ turn $_{i} \vee P \diamond_{a}^{-1}$ turn $\left._{i}\right)$.

To prove the converse, fix an action-labeled extensive form that violates property $(A R)$. Then there exist a player $i$, nodes $t, x, y$ and $y^{\prime}$, a choice $c$ and an action $a$ such that

$$
\begin{gathered}
t, y \in X_{i}, t \rightarrow_{c} x, \alpha(c)=a \text { and } x \precsim y, \\
y \sim_{i} y^{\prime}, \\
\forall t^{\prime}, x^{\prime}, c^{\prime} \text {, if } t^{\prime} \in X_{i}, t^{\prime} \rightarrow_{c^{\prime}} x^{\prime} \text { and } \alpha\left(c^{\prime}\right)=a \text {, then } x^{\prime} \neq y^{\prime} \text { and } x^{\prime} \nprec y^{\prime} .
\end{gathered}
$$

By (2),

$$
x \models \diamond_{a}^{-1} \text { turn }_{i}
$$

By (4), $y^{\prime} \not \models \diamond_{a}^{-1}$ turn $_{i} \vee P \diamond_{a}^{-1}$ turn $_{i}$. Thus, by (3),

$$
y \not \models K_{i}\left(\diamond_{a}^{-1} \text { turn }_{i} \vee P \diamond_{a}^{-1} \text { turn }_{i}\right) .
$$

If $x=y$, then by (5) and (6), axiom $\left(M E M_{A}\right)$ is falsified at $y$. If, on the other hand, $x \prec y$, then by (6), $x \not \models G K_{i}\left(\diamond_{a}^{-1}\right.$ turn $_{i} \vee P \diamond_{a}^{-1}$ turn $\left._{i}\right)$ and this, together with (5), falsifies $\left(M E M_{A}\right)$ at $x$.

\section{Axiomatization of perfect recall}

The two properties discussed in the previous sections, namely memory of past knowledge (property $(K M))$ and memory of past actions (property $(A R))$ are individually compatible with violations of perfect recall. For example, both the extensive form of Figure 2 and 
that of Figure 7 violate perfect recall. However, the former satisfies $(K M)$ (although it violates $(A R)$ ), while the latter satisfies $(A R)$ (although it violates $(K M)$ ). In this section we show that, together, they are equivalent to perfect recall. Thus the conjunction of the two axioms $\left(M E M_{K}\right)$ and $\left(M E M_{A}\right)$ provides a characterization of perfect recall and captures its traditional interpretation (suggested by Kuhn himself) as requiring players to remember both what they knew in the past and what they did.

Proposition 9 The conjunction of axioms $\left(M E M_{K}\right)$ and $\left(M E M_{A}\right)$ characterizes perfect recall, that is

(A) the axioms are valid in every action-labeled extensive form that satisfies perfect recall, and

(B) if both axioms are valid in an action-labeled extensive form, then the extensive form satisfies perfect recall.

The proof of Proposition 9 is based on the following lemmas. The first says that if property $(K M)$ holds, then the following is true for every player $i$ : if $y$ and $y^{\prime}$ are two nodes in the same information set of player $i$, then the sequence of information sets of player $i$ crossed by the path from the root to $y$ coincides with the sequence of information sets of player $i$ crossed by the path from the root to $y^{\prime}$. The second lemma says that, although property $(A R)$ by itself does not imply that a player remembers the order in which she took past actions (cf. Figure 7 ), once it is coupled with property $(K M)$ then the implication holds.

Lemma 10 Fix an extensive form that satisfies property $(K M)$. Fix a player $i$ and arbitrary $y, y^{\prime} \in X_{i}$ such that $y \sim_{i} y^{\prime}$. Let $\left\langle y_{1}, \ldots, y_{m}\right\rangle$ be the sequence of nodes obtained by removing from the path from the root to $y$ the nodes that do not belong to $X_{i}$ (thus (a) $y_{m}=y$, (b) for all $j=1, \ldots, m-1, y_{j} \in X_{i}$ and $y_{j} \prec y_{j+1}$, and (c) if $x \in X_{i}$ is such that $x \prec y$ then $x=y_{j}$ for some $j=1, \ldots, m-1)$. Similarly, let $\left\langle y_{1}^{\prime}, \ldots, y_{r}^{\prime}\right\rangle$ be the sequence of nodes obtained by 
removing from the path from the root to $y^{\prime}$ the nodes that do not belong to $X_{i}$. Then $m=r$ and, for all $j=1, \ldots, m, y_{j} \sim_{i} y_{j}^{\prime}$.

Proof. Fix an extensive form that satisfies property $(K M)$. By hypothesis, $y_{m} \sim_{i} y_{r}^{\prime}$ since $y_{m}=y$ and $y_{r}^{\prime}=y^{\prime}$. If $r=m=1$, there is nothing to prove. Suppose first that $m>1$ and $r=1$ (that is, no predecessor of $y^{\prime}$ belongs to $X_{i}$ ). Then $y_{m-1}, y_{m} \in X_{i}, y_{m-1} \prec y_{m}$ and $y_{m} \sim_{i} y_{r}^{\prime}$. By $(K M)$ there exists an $x \in X_{i}$ such that $y_{m-1} \sim_{i} x$ and $x \prec y_{r}^{\prime}$, yielding a contradiction. The case $m=1$ and $r>1$ is ruled out similarly. Suppose therefore that $m>1$ and $r>1$. First we show that $r \geq m$. For every $j=1, \ldots, m-1$ we have that $y_{j} \prec y_{m}$ and $y_{m} \sim_{i} y_{r}^{\prime}$. Thus by $(K M)$ there exists an $x_{j} \in X_{i}$ such that $y_{j} \sim_{i} x_{j}$ and $x_{j} \prec y_{r}^{\prime}$. Then $\left\{x_{1}, \ldots, x_{m-1}\right\} \subseteq\left\{y_{1}^{\prime}, \ldots, y_{r-1}^{\prime}\right\}$. Hence $r \geq m$. A symmetric argument shows that $m \geq r$ and therefore $r=m$. Next we show that $y_{m-1} \sim_{i} y_{m-1}^{\prime}$. By $(K M)$ there is a $j<m$ such that $y_{m-1} \sim_{i} y_{j}^{\prime}$. Consider the sequences $\left\langle y_{1}, \ldots, y_{m-1}\right\rangle$ and $\left\langle y_{1}^{\prime}, \ldots, y_{j}^{\prime}\right\rangle$. Since $y_{m-1} \sim_{i} y_{j}^{\prime}$ we can apply the previous argument and conclude that $m-1=j$. A repetition of this argument shows that $y_{j} \sim_{i} y_{j}^{\prime}$ for all $j=1, \ldots, m$.

Lemma 11 Fix an action-labeled extensive form that satisfies properties $(K M)$ and $(A R)$.

Fix a player $i$ and arbitrary $y, y^{\prime} \in X_{i}$ such that $y \sim_{i} y^{\prime}$. Let $\left\langle c_{1}, \ldots, c_{m-1}\right\rangle(m \geq 1)$ be the sequence of choices made by player $i$ along the path from the root to $y \cdot{ }^{13}$ Similarly, let $\left\langle c_{1}^{\prime}, \ldots, c_{r-1}^{\prime}\right\rangle$ be the sequence of choices made by player $i$ along the path from the root to $y^{\prime}$. Then $m=r$ and, for all $j=1, \ldots, m-1, c_{j}=c_{j}^{\prime}$.

Proof. Fix an action-labeled extensive form that satisfies properties $(K M)$ and $(A R)$. Let $\left\langle y_{1}, \ldots, y_{m-1}, y\right\rangle$ and $\left\langle y_{1}^{\prime}, \ldots, y_{r-1}^{\prime}, y^{\prime}\right\rangle$ be the sequences in $X_{i}$ defined in the statement of Lemma 10. Thus $c_{j}$ is the choice of player $i$ at $y_{j}$ (and, similarly, $c_{j}^{\prime}$ is the choice at $y_{j}^{\prime}$ ). By Lemma 10, $r=m$. The proof that, for all $j=1, \ldots, m-1, c_{j}=c_{j}^{\prime}$ is by induction. First we show that $c_{1}=c_{1}^{\prime}$. By definition of $y_{1}$ and $c_{1}, y_{1} \rightarrow_{c_{1}} x$ and $x \precsim y_{2}$, for some node $x$. By Lemma 10, $y_{2} \sim_{i} y_{2}^{\prime}$. Thus by $(A R)$ there exist $t^{\prime} \in X_{i}, c^{\prime}$ and $x^{\prime}$ such that $t^{\prime} \rightarrow_{c^{\prime}} x^{\prime}$, $x^{\prime} \precsim y_{2}^{\prime}$ and $\alpha\left(c^{\prime}\right)=\alpha\left(c_{1}^{\prime}\right)$. Since the only predecessor of $y_{2}^{\prime}$ that belongs to $X_{i}$ is $y_{1}^{\prime}$, it follows that $t^{\prime}=y_{1}^{\prime}$ and $c^{\prime}=c_{1}^{\prime}$. By Lemma 10, $y_{1} \sim_{i} y_{1}^{\prime}$. Thus, since $\alpha\left(c_{1}\right)=\alpha\left(c_{1}^{\prime}\right)$ and $y_{1}$ and $y_{1}^{\prime}$ belong to the same information set (of player $i$ ), it follows that $c_{1}=c_{1}^{\prime}$ (recall that, by definition, the function $\alpha(\cdot)$ must assign different action labels to different choices at the same information set). Next we prove the induction step. Suppose that it is true that $c_{j}=c_{j}^{\prime}$ for all $j=1, \ldots, r$ with $r \leq m-2$. We want to show that $c_{r+1}=c_{r+1}^{\prime}$. By definition

\footnotetext{
${ }^{13}$ That is, if $\left\langle y_{1}, \ldots, y_{m}\right\rangle$ is the sequence in $X_{i}$ defined in Lemma 10, then for every $j=1, \ldots, m-1, c_{j}$ is the choice at $y_{j}$ that lies on the path from the root to $y_{m}=y$.
} 
of $y_{r+1}$ and $c_{r+1}, y_{r+1} \rightarrow_{c_{r+1}} x$ and $x \precsim y_{r+2}$, for some node $x$. By Lemma 10, $y_{r+2} \sim_{i} y_{r+2}^{\prime}$. Thus by $(A R)$ there exist $t^{\prime} \in X_{i}, c^{\prime}$ and $x^{\prime}$ such that $t^{\prime} \rightarrow_{c^{\prime}} x^{\prime}, x^{\prime} \precsim y_{r+2}^{\prime}$ and

$$
\alpha\left(c^{\prime}\right)=\alpha\left(c_{r+1}\right)
$$

Since $t^{\prime} \in X_{i}, t^{\prime}=y_{k}^{\prime}$ for some $k \leq r+1$. We want to show that $k=r+1$. Suppose, by contradiction, that $k<r+1$. Then $c^{\prime}=c_{k}^{\prime}$ and, by (7), $\alpha\left(c_{k}^{\prime}\right)=\alpha\left(c_{r+1}\right)$. By the induction hypothesis, $c_{k}^{\prime}=c_{k}$. Thus $\alpha\left(c_{k}\right)=\alpha\left(c_{r+1}\right)$ contradicting the property of the function $\alpha(\cdot)$ which rules out the same action label for two sequential choices of the same player at different information sets. Thus $t^{\prime}=y_{r+1}^{\prime}$ and $c^{\prime}=c_{r+1}^{\prime}$. By Lemma 10, $y_{r+1} \sim_{i} y_{r+1}^{\prime}$. Thus, by (7), $\alpha\left(c_{r+1}^{\prime}\right)=\alpha\left(c_{r+1}\right)$ and, since $y_{r+1}$ and $y_{r+1}^{\prime}$ belong to the same information set, we must have that $c_{r+1}^{\prime}=c_{r+1}$.

Proof of Proposition 9. By Propositions 3 and 8, it is enough to show that perfect recall $(P R)$ is equivalent to the conjunction of $(K M)$ and $(A R)$. That $(P R)$ implies both $(K M)$ and $(A R)$ is straightforward. Thus we shall prove only the converse. Fix an actionlabeled extensive form that satisfies both $(K M)$ and $(A R)$. Fix arbitrary player $i$, nodes $t, y, y^{\prime} \in X_{i}$ and $x \in T$ and a choice $c$ such that $t \rightarrow_{c} x, x \precsim y$ and $y \sim_{i} y^{\prime}$. By Lemma 11, the sequence of choices of player $i$ from the root to $y$ coincides with the sequence of choices of player $i$ from the root to $y^{\prime}$. Thus, since choice $c$ precedes $y$, there exist nodes $t^{\prime} \in X_{i}$ and $x^{\prime} \in T$ such that $t^{\prime} \rightarrow_{c} x^{\prime}$ and $x^{\prime} \precsim y^{\prime}$. Since $t \rightarrow_{c} x$ and $t^{\prime} \rightarrow_{c} x^{\prime}$, by definition of choice (a set of edges at the same information set), it must be that $t \sim_{i} t^{\prime}$.

\section{Related literature}

Some of the issues discussed in this paper are relevant to, and have been studied in, different fields. In this section we review related contributions in game theory, computer science and logic.

A. Game theory literature. Several characterizations of perfect recall have been proposed in game theory. Okada (1987, Proposition 4, p. 89) shows that, within the class of extensive forms that satisfy property $(K M)$, perfect recall is equivalent to complete inflation (see also Ritzberger, 1999, Theorem 2(b), p. 81). ${ }^{14}$ Since, by Proposition 9,

\footnotetext{
${ }^{14} \mathrm{An}$ extensive form is completely inflated if there is no information set that contains an isolated subset. A subset $v$ of an information set $h$ of player $i$ is isolated in $h$ if, for every two nodes $y \in v$ and $y^{\prime} \in h \backslash v$,
} 
within the class of extensive forms that satisfy $(K M)$, perfect recall is equivalent to property $(A R)$, it follows that, within this class, action recall is equivalent to complete inflation. Outside this class, however, the equivalence breaks down: $(A R)$ and complete inflation are distinct and independent properties. The extensive form of Figure 7 satisfies $(A R)$ but violates complete inflation (since $\{x\}$ is an isolated subset of $\{x, y\}$ ), while the extensive form of Figure 8 is completely inflated but violates $(A R)$. Several alternative semantic characterizations of perfect recall, involving properties such as complete inflation, weak and strong recall, etc., are provided by Ritzberger (1999, Theorem 2, p. 81).

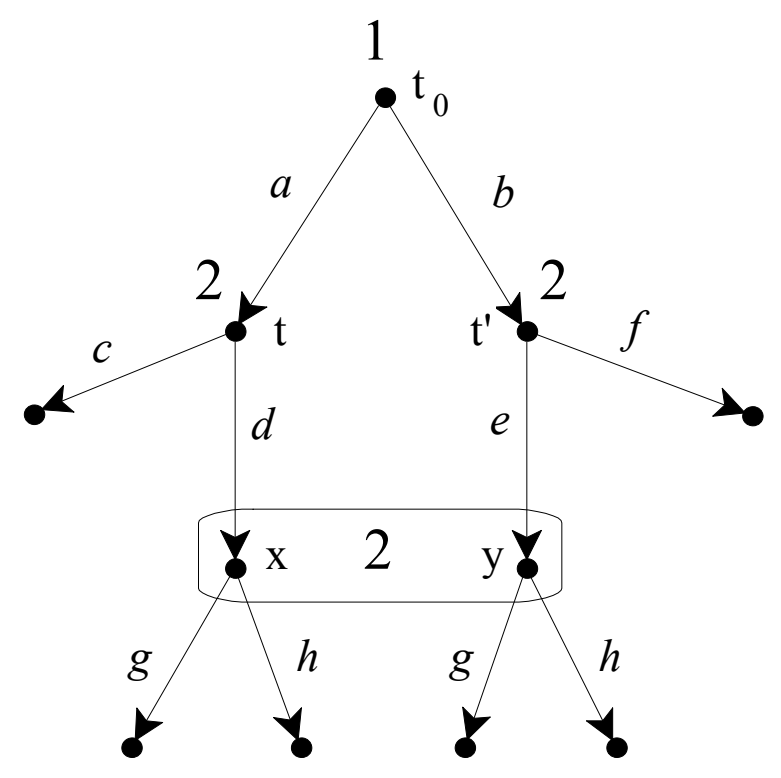

Figure 8

B. Computer science literature. The interaction of knowledge and time has been studied extensively in computer science. In particular, the property that we called 'memory of past knowledge' was introduced by Ladner and Reif (1986) and Halpern and Vardi (1986). In the latter this property was called 'no forgetting'. It was later

there exists another information set $h^{\prime}$ of player $i$ and two distinct choices $c$ and $c^{\prime}$ at $h^{\prime}$ such that $y$ comes after choice $c$ and $y^{\prime}$ comes after choice $c^{\prime}$. 
renamed as 'perfect recall' in Fagin et al. (1995). Within the context of systems of runs, where the knowledge of every agent $i$ is specified at every instant (unlike extensive forms, where the knowledge of a player is specified only at nodes where it is his turn to move) Halpern and Vardi (1986) proposed the following axiom to capture the property of no forgetting: $K_{i} \square \phi \rightarrow \square K_{i} \phi$, where $\square \phi$ stands for $(\phi \wedge G \phi)$, that is, $\square \phi$ means ' $\phi$ is true now and at every future moment'. Adapted to the context of extensive forms, this axiom can be restated as follows: $\left(\operatorname{turn}_{i} \wedge K_{i} \square \phi\right) \rightarrow \square K_{i} \phi$. It can be shown that this axiom provides an alternative characterization of property $\left(M E M_{K}\right)$. Halpern and Vardi (1986) also provide a sound and complete axiomatization of systems that satisfy 'no forgetting' and are synchronous (i.e. the agents have access to an external clock). The key axiom is $K_{i} \bigcirc \phi \rightarrow \bigcirc K_{i} \phi$, where $\bigcirc$ is the 'next time' operator, that is, $t=\bigcirc \phi$ if $\phi$ is true at every immediate successor of $t$. A thorough account of sound and complete axiomatizations of systems where knowledge and time interact is given in Halpern et al. (2002). Synchronous systems are closely related to von Neumann games (see the discussion in Section 3 concerning axiom $\left(M E M_{K}^{\prime}\right)$ and the remarks in the next paragraph).

C. Logic literature. Van Benthem (2001) looks at extensive games as models for a joint dynamic-epistemic language. Significant conditions on those games are shown to be definable by certain axioms in that combined language. In particular, van Benthem proposes the following axiom to capture the property of perfect recall: $\left(\operatorname{turn}_{i} \wedge K_{i} \square_{c} \phi\right) \rightarrow$ $\square_{c} K_{i} \phi$, where $c$ denotes a choice and the modal operator $\square_{c}$ is analogous to our operator $\square_{a}$ for actions. Thus $\square_{c} \phi$ is true at a decision node $t$ if $\phi$ is true at the immediate successor of node $t$ following choice $c$. It can be shown that this axiom is valid in every von Neumann extensive form. Recall that an extensive form is von Neumann if any two nodes that belong to the same information set have the same number of 
predecessors. ${ }^{15}$ The axiom proposed by van Benthem, however, does not provide a general characterization of perfect recall, since there are extensive forms that satisfy perfect recall where the axiom is not valid. Consider, for example, the extensive form of Figure 1, which satisfies perfect recall. Let $q$ be an atomic proposition and construct a model where the truth set of $q$ is $\{x, y\}$. The model is shown in Figure 9, where only the relevant portion of the tree is highlighted and the formulas that are true at a node are written next to it. Since $V(q)=\{x, y\}, \square_{d} q$ is true at both $t$ and $t^{\prime}$ and therefore at $t$ player 2 knows that $\square_{d} q: t \models K_{2} \square_{d} q$. Furthermore, since $t \in X_{2}, t \models$ turn $_{2}$. Thus $t \models$ turn $_{2} \wedge K_{2} \square_{d} q$. Since $q$ is false at $x^{\prime}$ and $x \sim_{2} x^{\prime}$, it is not the case that player 2 knows that $q$ at $x: x \not \models K_{2} q$. Thus, since $t \rightarrow_{d} x, t \not \models \square_{d} K_{2} q$. Thus we have constructed a model where the axiom is falsified at node $t$.

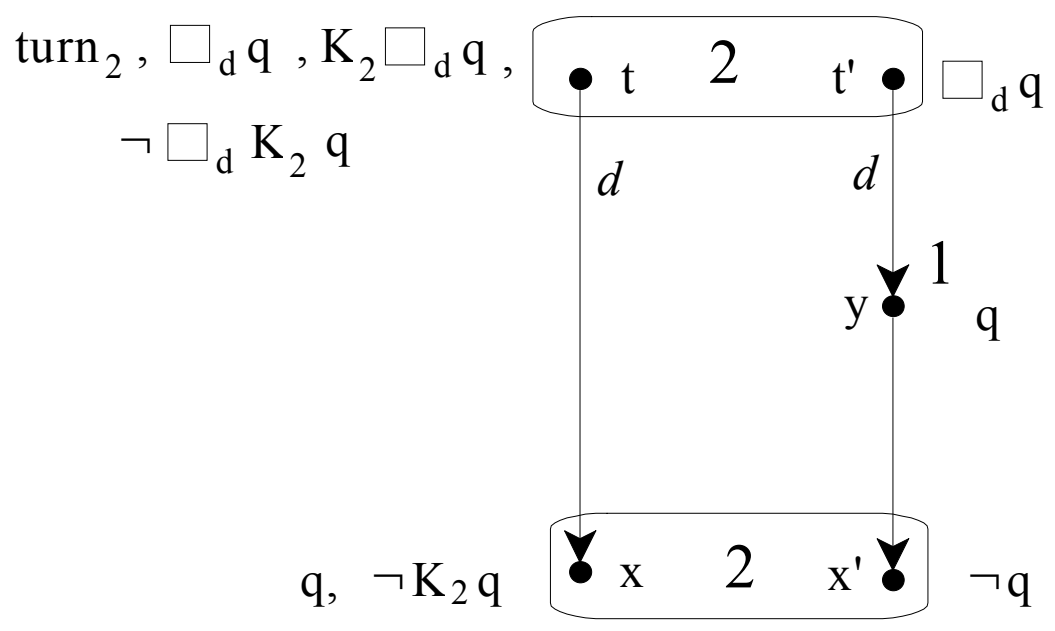

Figure 9

\footnotetext{
${ }^{15}$ That is, if $x \sim_{i} t$ then the length of the path from the root to $x$ is equal to the length of the path from the root to $t$. The length of the path to a node can be interpreted as the number of units of time that have elapsed since the beginning of the game. Thus in a von Neumann game a player always 'knows the time' when it is her turn to move. Hence von Neumann games are closely related to the synchrounous systems studied by Halpern and Vardi (1986). The expression 'von Neumann games' was introduced by Kuhn (1953).
} 


\section{Conclusion}

We decomposed perfect recall into two independent properties: memory of past knowledge and memory of past actions. For each property we provided a characterizing axiom and showed that the conjunction of the two is equivalent to perfect recall. Thus we obtained an axiomatic basis for the traditional interpretation of perfect recall, suggested by Kuhn himself, as "equivalent to the assertion that each player is allowed by the rules of the game to remember everything he knew at previous moves and all of his choices at those moves". Since, in general, the set of actions yields a coarser partition than the choice partition, our characterization actually shows that the last part of Kuhn's claim is more than what is needed: it is sufficient for the player to remember what actions she took in the past and not necessarily what choices.

\section{References}

[1] van Benthem, J. (2001), Games in dynamic epistemic logic, Bulletin of Economic Research, 53, 219-248.

[2] Blackburn, P., M. de Rijke and Y. Venema (2001), Modal logic, Cambridge University Press.

[3] Bonanno, G. (2001), Memory requires von Neumann games, Working Paper, Department of Economics, University of California, Davis.

[4] Burgess, J. (1984), Basic tense logic, in: D. Gabbay and F. Guenthner (eds.), Handbook of philosophical logic, Vol. II, D. Reidel Publishing Company, 89-133.

[5] Chellas, B. (1984), Modal logic: an introduction, Cambridge University Press.

[6] Dalkey, N. (1953), Equivalence of information patterns and essentially determinate games, Annals of Mathematical Studies, 28, 217-243.

[7] Fagin, R, J. Halpern, Y. Moses and M. Vardi (1995), Reasoning about knowledge, MIT Press.

[8] Goldblatt, R. (1992), Logics of time and computation, CSLI Lecture Notes No. 7. 
[9] Halpern, J. (1998), Characterizing the common prior assumption, in: I. Gilboa, Ed., Theoretical aspects of rationality and knowledge: Proceedings of the seventh conference, Morgan Kaufmann, 133-146. (To appear in Journal of Economic Theory.)

[10] Halpern, J. and M. Vardi (1986), The complexity of reasoning about knowledge and time, Proceedings 18th ACM Symposium on Theory of Computing, 304-315.

[11] Halpern, J., R. van der Meyden and M. Vardi (2002), Complete axiomatizations for reasoning about knowledge and time, Working Paper, Cornell University. (To appear in SIAM Journal on Computation.)

[12] Kline, J. J. (2002), Minimum memory for equivalence between ex ante optimality and time consistency, Games and Economic Behavior, 38, 278-305.

[13] Kuhn, H. W. (1953), Extensive games and the problem of information, in: H. W. Kuhn and W. W. Tucker (eds.), Contributions to the theory of games, Vol. II, Princeton University Press, 193-216. Reprinted in Kuhn (1997), 46-68.

[14] Kuhn, H. W. (1997), Classics in game theory, Princeton University Press.

[15] Ladner, R. and J. Reif (1986), The logic of distributed protocols (preliminary report), in: J. Halpern, Ed., Theoretical aspects of reasoning about knowledge: Proceedings of the 1986 conference, Morgan Kaufmann, 207-222.

[16] Okada, A. (1987), Complete inflation and perfect recall in extensive games, International Journal of Game Theory, 16, 85-91.

[17] Piccione, M. and A. Rubinstein (1997), On the interpretation of decision problems with imperfect recall, Games and Economic Behavior, 20, 3-24.

[18] Ritzberger, K. (1999), Recall in extensive form games, International Journal of Game Theory, 28, 69-87.

[19] Ritzberger, K. (2002), Foundations of non-cooperative game theory, Oxford University Press.

[20] Selten, R. (1975), Re-examination of the perfectness concept for equilibrium points in extensive games, International Journal of Game Theory, 4, 25-55. Reprinted in Kuhn (1997), 317-354. 